\title{
A Non-Markovian Multistage Batch Arrival Queue with Breakdown and Reneging
}

\author{
Sivagnanasundararam Maragathasundari ${ }^{1,2}$ and Santhanagopalan Srinivasan ${ }^{3}$ \\ ${ }^{1}$ Sathyabama University, Chennai 600 119, India \\ ${ }^{2}$ Department of Mathematics, Velammal Institute of Technology, Chennai 601 204, India \\ ${ }^{3}$ Department of Mathematics, B. S. Abdur Rahman University, Chennai 600 048, India
}

Correspondence should be addressed to Sivagnanasundararam Maragathasundari; maragatham01@gmail.com

Received 21 July 2014; Accepted 7 October 2014; Published 13 November 2014

Academic Editor: Antonina Pirrotta

Copyright (C) 2014 S. Maragathasundari and S. Srinivasan. This is an open access article distributed under the Creative Commons Attribution License, which permits unrestricted use, distribution, and reproduction in any medium, provided the original work is properly cited.

\begin{abstract}
The present investigation deals with analysis of non-Markovian queueing model with multistage of services. When the server is unavailable during the system breakdown (or) vacation periods, we consider reneging to prevail. Supplementary variable techniques have been adopted to obtain steady state system length distributions. The numerical illustrations are provided to validate the tractability of performance measures as far as computational aspect is concerned. Numerical results in the form of graphical representation are also presented. Practical large scale industry applications are described to justify our model.
\end{abstract}

\section{Introduction}

Vacation queueing models play a major role in manufacturing and production, computer and communication, and service and distribution systems. Many models for customer's impatience in queueing systems have been studied in the past, and the source of impatience has always been considered to be either a long wait already experienced at a queue or a long wait anticipated by a customer upon arrival. Queueing models enable organizations to implement critical production strategies or tactics aimed at reducing costs on increasing revenues. By examining the methods of operations research and especially queueing theory, new models can be developed and existing ones can be extended.

One of the earliest works on balking and reneging was by Haight $[1,2]$ and Barrer [3], which was the first to introduce reneging in which they studied deterministic reneging with single server Markovian arrival and service rates. MontazerHaghighi et al. [4] studied a Markovian mutiserver queueing system with balking and reneging. A two-stage batch arrival queueing system where customers receive a batch service in the first and individual service in the second stage was studied by Doshi [5] in the past.
Furthermore [6], Chodhury examined an $\mathrm{M}^{[x]} / \mathrm{G} / 1$ queueing system with a set-up period and a vacation period. This paper deals with an $\mathrm{M}^{X} / \mathrm{G} / 1$ queueing system with a vacation period which comprises an idle period and a random set-up period. The server is turned off each time when the system becomes empty. At this point of time the idle period starts. As soon as a customer or a batch of customers arrive, the setup of the service facility begins which is needed before starting each busy period. In this paper the steady-state behaviour of the queue size distributions at stationary (random) point of time and at departure point of time is studied. Also, explicit expressions for the system state probabilities and some performance measures of this queueing system are derived analytically. Finally, the probability generating function of the additional queue size distribution due to the vacation period as the limiting behaviour of the $\mathrm{M}^{X} / \mathrm{M} / 1$ type queueing system is derived.

Madan [7] investigated a batch arrival queueing system, where the server provides two stages of heterogeneous service with a modified Bernoulli schedule under $N$-policy. The server remains idle till the queue size becomes $N(\geq 1)$. As soon as the queue size becomes at least $N$, the server instantly starts working and provides two stages of service 
in succession to each customer, that is, the first stage service followed by the second stage service. However, after the second stage service, the server may take a vacation or decide to stay in the system to provide service to the next customer, if any. The queue size distributions at a random epoch as well as a departure epoch under the steady-state conditions were derived.

The M/G/1 queue with impatient customers was studied by Bae et al. [8]; the complete formula of the limiting distribution of the virtual waiting time was derived explicitly. The expected busy period of the queue was also obtained by using a martingale argument.

In 2002, Krishna Kumar et al. [9] studied an M/G/1 retrial queueing system with two-phase service and preemptive resume. In this paper, for an arbitrarily distributed retrial time distribution, the necessary and sufficient condition for the system stability is obtained, assuming that only the customer at the head of the orbit has priority access to the server. The steady-state distributions of the server state and the number of customers in the orbit are obtained along with other performance measures. The effects of various parameters on the system performance are analysed numerically. A general decomposition law for this retrial queueing system is established.

Furthermore Madan et al. [10] studied the server vacations based on Bernoulli schedules and a single vacation policy in 2003. In this model, a single server queue with optional server vacations based on exhaustive service is studied. Unlike other vacation policies, the assumption that, only at the completion of service of the last customer in the system, the server has the option to take a vacation or to remain idle in the system waiting for the next customer to arrive is considered. The service times of the customers have been assumed to be deterministic and vacations are phase type exponential. Steady-state results for the probability generating functions of the queue length, the expected number of customers in the queue, and the expected waiting time of the customer are derived explicitly.

In addition, Choudhury [11] investigated a batch arrival queueing system with an additional service channel. This paper deals with an $\mathrm{M}^{x} / \mathrm{G} / 1$ queueing system with two phases of heterogeneous service under $N$-policy, where the server remains idle till the queue size becomes $N(\geqslant 1)$. As soon as the queue size becomes $N$, the server immediately starts first "essential service" for all the units. After completion of the essential service of a unit, it may leave the system with probability $(1-\theta)$ or may immediately go for a second phase of service in an additional service channel with probability $\theta$ $(0 \leqslant \theta \leqslant 1)$. For this model, the queue size distribution at a random epoch as well as at a departure epoch is obtained. This is a generalization of recent papers considered by Medhi and Lee et al. (queueing systems).

In 2004 Madan and Abu Al-Rub [12] investigated on a single server queue with optional phase type server vacations based on exhaustive deterministic service and a single vacation policy. Furthermore Madan and Choudhury [13] proposed a queueing system with restricted admissibility of arriving batches. In this model a batch arrival queue with a Bernoulli vacation schedule is considered. After completion of the service the server either goes for a vacation in random length with probability $\theta \quad(0 \leq \theta \leq 1)$ or may continue to serve the next unit, if any, with probability $(1-\theta)$ under a restricted admissibility policy of arriving batches. Unlike the usual batch arrival queueing system the restricted admissibility policy differs during a busy period and a vacation period and hence all arriving batches are not allowed to join the system at all time. Steady-state queue size distribution at a random point of time as well as a departure epoch is derived. Moreover this paper attempts to unify several classes of related batch arrival queueing system.

Choudhury and Madan [14] studied a two-stage batch arrival queueing system with a modified Bernoulli schedule vacation under $N$-policy. In this paper a batch arrival queueing system, where the server provides two stages of heterogeneous service with a modified Bernoulli schedule under $N$-policy, is considered. The server remains idle till the queue size becomes $N(\geq 1)$. As soon as the queue size becomes at least $N$, the server instantly starts working and provides two stages of service in succession to each customer, that is, the first stage service followed by the second stage service. However, after the second stage service, the server may take a vacation or decide to stay in the system to provide service to the next customer, if any. The queue size distribution at a random epoch as well as a departure epoch under the steady-state conditions is derived. Further, the existence of the stochastic decomposition property to show that the departure point queue size distribution of this model can be decomposed into the distributions of three independent random variables is demonstrated. Finally, a simple procedure to obtain optimal stationary operating policy under a suitable linear cost structure is developed.

Furthermore Chang and Takine [15] studied factorization and stochastic decomposition properties in bulk queues with generalized vacation. This paper considers a class of stationary batch-arrival, bulk-service queues with generalized vacations. The system consists of a single server and a waiting room of infinite capacity. Arrivals of customers follow a batch Markovian arrival process. The server is unavailable for occasional intervals of time called vacations, and, when it is available, customers are served in groups of fixed size B. For this class of queues, the vector probability generating function of the stationary queue length distribution is factored into two terms, one of which is the vector probability generating function of the conditional queue length distribution given that the server is on vacation is shown. The special case of batch Poisson arrivals is examined and a new stochastic decomposition formula is derived for the stationary queue length distribution.

Altman and Yechiali [16] considered the systems with servers vacations where customers' impatience is due to an absentee of servers upon arrival. Such a model, representing frequent behavior by waiting customers in service systems, had never been treated before in the literature.

Altman and Yechiali [17] studied a system that is operating as an $\mathrm{M} / \mathrm{M} / \infty$ queue. However, when it becomes empty, it is assigned to perform another task, the duration $U$ of which is random. Customers arriving while the system is unavailable for service (i.e., occupied with a U-task) become 
impatient: each individual activates an "impatience timer" having random duration $\mathrm{T}$ such that if the system does not become available by the time the timer expires, the customer leaves the system never to return. When the system completes a U-task and there are waiting customers, each one is taken immediately into service. Explicit expressions for the corresponding mean queue sizes were obtained.

In 2012, Kumar and Sharma [18] analyzed a single server queue with general service time distribution, random system breakdowns, and Bernoulli schedule server vacations where, after a service completion, the server may decide to leave the system with probability $p$ or to continue serving customers with probability $1-p$. It is assumed that the customers arrive to the system in batches of variable size but are served one by one. If the system breaks down, it enters a repair process immediately. It is assumed that the repair time has general distribution, while the vacation time has exponential distribution. The purpose is to find the steady-state results in explicit and closed form in terms of the probabilitygenerating functions for the number of customers in the queue, the average number of customers, and the average waiting time in the queue.

Also Maraghi et al. [19] studied an $\mathrm{M}^{x} / \mathrm{G} / 1$ queue with random breakdowns and Bernoulli schedule server vacations where, after a service completion, the server may decide to leave the system with probability $p$ or to continue serving customers with probability $1-p$. It is assumed that customers arrive to the system in batches of variable size but are served one by one.

Kumar and Sharma [20] analysed the concept of customer balking and reneging has been exploited to a great extent in the recent past by the queuing modelers. According to this model, a reneged customer can be convinced in many cases by employing certain convincing mechanism to stay in the queue for completion of his service. Thus, a reneged customer can be retained in the queuing system with some probability (say, $q$ ) and it may leave the queue without receiving service with probability $p(=1-q)$. This process was referred to as customer retention. The effect of probability of retention on the average system size had been studied.

Recently in 2013, Baruah et al. [21] studied a two-stage queuing model where the server provides two stages of service one by one in succession. They considered reneging to occur when the server is unavailable during the system breakdown or vacation periods.

Kumar and Sharma [22] in their queueing model with retention of reneged customers and balking studied a finite capacity and the retention of reneged customers. The customer's impatience may occur in the form of balking also. In this paper, the work of Kumar and Sharma (2013) is extended by including balking to take into consideration the broader perspective of customer's impatience. The model is solved iteratively to obtain the steady-state probabilities of system size. Some useful performance measures are derived, and the effect of the probability of retaining the reneged customers on various performance measures is studied numerically.
1.1. Model Description. In today's competitive world, customer's impatience has become a serious problem. Firms are employing a number of strategies to retain their customers. To the best of our knowledge, studies on multistage batch arrival of service in reneging do not exist. We extend and develop the above models on two stages of service to multistage of services. In particular, a two-stage model of Monita Baruah, Kailash C. Madan, and Tillal Eldabi in the year 2013 motivated us for developing this new model which we consider here. Their model on two stages of service is extended to multistage of service where the arrivals occur in batches. Breakdown and reneging play a vital role in our model. Two stages of service will be benefited only for small scale industries, factories of low production, limited network, and so forth, whereas multistages of services render a huge amount of beneficial factors in large scale industries and in multitier applications in e-commerce in particular.

This results in an introduction of a new model: a nonMarkovian multistage batch arrival queue with breakdown and reneging. In this model once the customers enter they will be provided with the service in all the stages of service department one by one in succession. Once the service gets completed, customer departs from the system. Once the multistage service of a unit is completed, the server is assumed to take vacation with probability $\theta$ or may continue to offer service with probability $(1-\theta)$. As soon as the vacation period of the server ends, it joins the system to continue service of the waiting customers. During system breakdown (or) vacation periods, there will be unavailability of service; in that case reneging occurs. Reneging is assumed to follow exponential distribution. Arrival follows a Poisson process and service time follows general distribution. We study the changes in the probability of idle time and in the traffic intensity in some particular cases like (1) if there is no vacation and (2) if the service time and vacation time are exponentially distributed. Using supplementary variable technique we derive the steady-state probabilities and performance measures.

This paper is organized as follows. Applications of model is presented in Section 2, the mathematical description of the model is given in Section 3, the definitions and notations are described in Section 4, the equations governing the system are given in detail using birth and death process in Section 5, the queue size distribution at random epoch is highlighted in Section 6, and the average queue size and average waiting time are given in Section 7. Sections 6 and 7 gives a comprehensive idea about the model in which step by step method is followed for better understanding which helps in any extension of this model. The particular cases are derived in Section 8 in which the model is seen in various aspects or situations. For justification of the model, the numerical illustrations are given in Section 9, and, at last, the conclusion is presented in Section 10. In this section future scope of this model is discussed. 


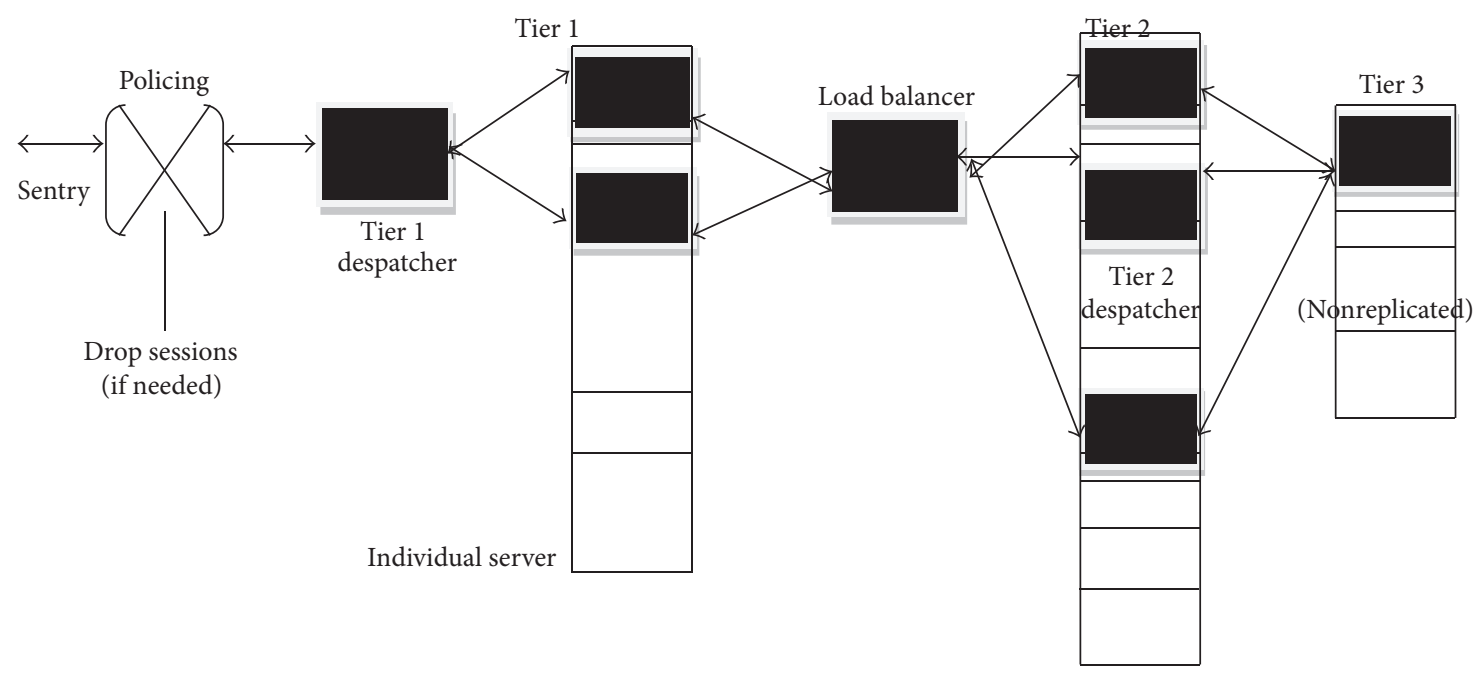

Figure 1: A three-tier application in e-commerce.

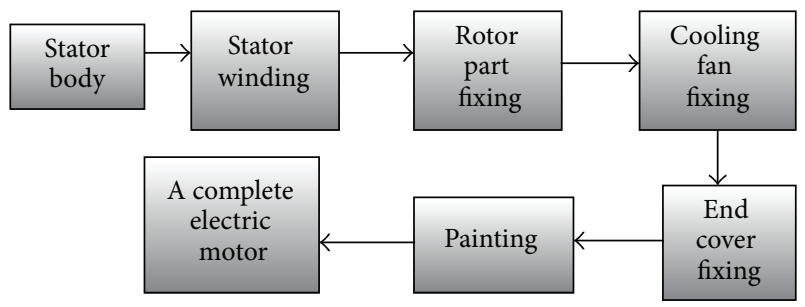

FIGURE 2: Multistage motor production process.

\section{Applications of Model}

With the development of computer and communication networks, queueing systems and networks have been identified as a powerful analysis and design tool for various applications. Our model plays a major role in multitier applications in e-commerce, motor production process, car production process, and textile industry. Some multistage real life applications in large scale manufacturing industry are described in the following sections which come across the necessity of our new model.

2.1. Three-Tier Application in e-Commerce. Consider a threetier application, as shown in Figure 1. The first two tiers are clustered web server and clustered java application, respectively, and the third tier is a database used in ecommerce applications. Each tier is considered to be a stage. Each incoming application is subjected to admission control at the sentry to ensure that the contracted performance guarantees are met. The excess sessions are turned away during overloads. The load balancer efficiently distributes the incoming requests. Each input data is considered to be a customer. The server maintenance period is considered to be a vacation period, and this application can be extended to multistage of services.

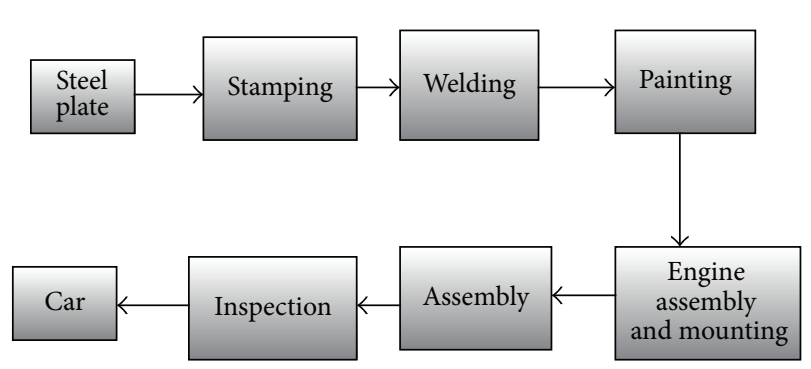

FIGURE 3: Multistage car production process.

\subsection{Electric Motor Production Industry}

Multistage in Motor Production Process (See Figure 2). In the electric motor manufacturing industries, multistage production process is implemented (Figure 2). The stator body is considered as the arrival customer. In the first stage, the stator body is wounded by the coil; in the second stage, the rotor part is fixed in the stator; in the third stage, the cooling fan is fixed in one end of the rotor; in the fourth stage, the end cover is fixed in the other end of the rotor; in the fifth stage, the entire motor is painted; and, finally, in the sixth stage, a complete electric motor is produced. We consider the stator body as a batch arrival customer. In the production sector, vacation denotes obtaining new raw material, maintenance of the tools, stock verification, and so forth. Here, the machine gets idle owing to breakdown, and the machine idle period is considered as the breakdown period.

\subsection{Automobile Industry}

Multistage in Car Production Process (See Figure 3). In automobile industry, car production process is considered as the multistage production process (Figure 3). Here, we consider the steel plate as the batch arrival customer. In the first stage of production, a roll of steel plate is cut according to the shape of the car parts in the second stage, and steel 


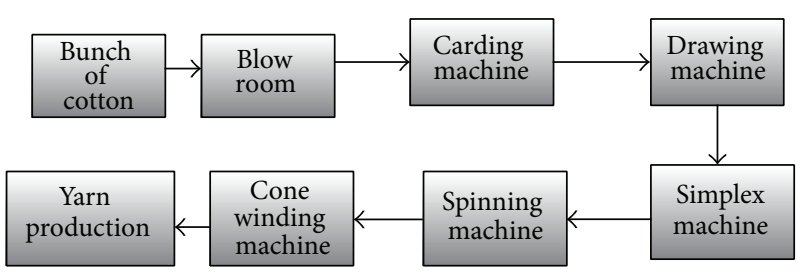

FIGURE 4: Textile yarn production process.

plates are welded; in the third stage, all the car parts are painted in the painting section; in the fourth stage, the engine assembly is mounted; in the fifth stage, extra fittings are assembled according to the customer requirements; in the sixth stage, quality control inspection is conducted; and, finally, a complete car is produced. We consider the steel plate as the batch arrival customer. In the production sector, vacation denotes obtaining new raw material, maintenance of the tools and machines, stock verification, and so forth. Here, machine gets idle owing to breakdown, and the machine idle period is considered as the breakdown period.

\subsection{Textile Yarn Production Industry}

Multistage in Production Process (See Figure 4). In the textile industry, yarn production is considered as the multistage production process (Figure 4). Here, a bunch of cotton is considered as the arrival customer. In the first stage, a bunch of cotton is inserted into the blow room machine; in the second stage, the refined cotton is inserted into the carding machine; in the third stage, the produced cotton sliver is inserted into the drawing machine and is again processed; in the fourth stage, the processed cotton sliver is inserted into the simplex machine; in the fifth stage, the refined cotton sliver is inserted into the spinning machine; and in the sixth stage, the cotton yarn output from spinning machine is inserted into the cone winding machine, and the entire cotton yarn is wounded as a cone. These six stages of production are required to convert a bunch of cotton into cotton yarn. In the production sector, vacation denotes obtaining a new raw material, maintenance of the tool and machine, stock verification, and so forth. Here, machine gets idle owing to breakdown, and the machine idle period is considered as the breakdown period.

\section{The Mathematical Description of the Model}

3.1. The Model Is Based on the Following Assumptions. (a) Customers arrive in batches following a compound Poisson process with a rate of arrival $\lambda$. Let $\lambda c_{i} d t(i=1,2,3, \ldots, n)$ be the first-order probability that customers arrive at the system in batches of size $i$ at the system in a short interval of time $(x, x+d t)$, where $0 \leq c_{i} \leq 1$ and $\sum_{i=1}^{\infty} c_{i}=1$.

(b) The server provides multistage of heterogeneous services one after the other in succession. An arrival batch receives the service offered at multistage in succession, defined as the first stage, second stage, and so forth, respectively. The service discipline is assumed to be on a first come first served basis (FCFS). Let us assume that the service time $S_{j}(j=1$ to $n)$ of the $j$ th stage service follows a general probability distribution with a distribution function $K_{j}\left(S_{j}\right)$, where $k_{j}\left(s_{j}\right)$ is the probability density function and $E\left(S_{j}^{n}\right)$ is the $n$th moment of the service time, with $j=1,2, \ldots, N$.

Let

$$
\begin{gathered}
\mu_{j}(x)=\frac{k_{j}(x)}{1-K_{j}(x)} \quad j=1,2, \ldots, N, \\
k_{j}\left(S_{j}\right)=\mu_{j}(x) e^{\left[-\int_{0}^{s} \mu_{j}(x) d x\right]}, \quad \text { where } j=1,2, \ldots, N .
\end{gathered}
$$

(c) Once the multistage service of a unit is completed, the server is assumed to take vacation with probability $\theta$ or may continue to offer service with probability $(1-\theta)$. As soon as the vacation period of the server ends, it joins the system to continue the service of the waiting customers.

Let us assume the vacation time to be a random variable following general probability law with distribution $M(v)$ and density function by $m(v) . E\left(V^{n}\right)$ denotes the $n$th moment $(n=1,2, \ldots)$ of the vacation time. Here, let us consider that $\xi(x)$ is the conditional probability of a vacation period during the interval $(x, x+d x)$, given that elapsed time is $x$, which can be given as

$$
\xi(x)=\frac{M(x)}{1-M(x)} .
$$

Thus,

$$
m(v)=\xi(v) e^{\left[-\int_{0}^{v} \xi(x) d x\right]} .
$$

(d) Customers arriving for service may become impatient and renege after joining during vacations and breakdown periods. Reneging is assumed to follow exponential distribution with parameter $\psi$. Thus, $f(t)=\psi e^{-\psi t} d t, \psi>0$. Thus $\psi d t$ is the probability that a customer can renege during a short interval of time $(t, t+d t)$.

The system may fail or be subjected to breakdown at random. The customer receiving service during breakdown returns back to the head of the queue. Let us assume that the interval between breakdowns occurs according to a Poisson process with a mean rate of breakdown $\beta>0$.

Furthermore, the repair times follow a general (arbitrary) distribution with the distribution function $G(x)$ and density function $g(x)$. Let the conditional probability of completion of the repair process be $\Upsilon(x) d x$ such that $\Upsilon(x)=$ $G(x) /(1-G(x))$, and, thus, $G(r)=\Upsilon(r) e^{\left(-\int_{0}^{r} \Upsilon(x) d x\right)}$.

\section{Definitions and Notations}

We assume that steady state exists and define $P_{n, j}(x)$ as the probability that there are $n(\geq 1)$ customers in the system including one customer in type $j$ service, $j=1,2, \ldots, N$, and elapsed service time is $x . P_{n, j}=\int_{0}^{\infty} P_{n, j}(x) d x$ is the corresponding steady-state probability irrespective of elapsed time $x . V_{n}(x)$ is the probability that there are $n(\geq 0)$ customers in the queue and server is on vacation and elapsed vacation time is $x$. 
$V_{n}=\int_{0}^{\infty} V_{n}(x) d x$ is the corresponding steady-state probability irrespective of elapsed vacation time $x$.

$Q$ is the steady-state probability of the server being idle as the server takes vacation.

The probability generating functions are defined as

$$
\begin{gathered}
P_{j}(x, z)=\sum_{n=1}^{\infty} z^{n} P_{n, j}(x) ; \\
P_{j}(x, z)=\sum_{n=1}^{\infty} z^{n} P_{n, j}|z| \leq 1 ; \quad j=1,2, \ldots, N, \\
R(x, z)=\sum_{n=1}^{\infty} z^{n} R_{n}(x) ; \\
R(z)=\sum_{n=1}^{\infty} z^{n} R_{n} ; \quad|z| \leq 1, \\
V(x, z)=\sum_{n=1}^{\infty} z^{n} V(x) ; \\
V(z)=\sum_{n=1}^{\infty} z^{n} V_{n} ; \quad|z| \leq 1, \\
C(z)=\sum_{i=1}^{\infty} z^{i} C_{i} .
\end{gathered}
$$

\section{Equations Governing the System}

We frame the difference equations with Poisson input related to birth-death process.

Let $n$ be the size of customers at time $x$. Let $P_{n}(x)$ be the probability of " $n$ " customers in the system.

Let

(1) $\lambda_{n}$ be the average arrival rate when " $n$ " customers are in the system;

(2) $\mu_{n}$ the average service rate when " $n$ " customers are in the system;

(3) $P_{n}(x+\Delta x)$ the probability of " $n$ " customers at $(x+\Delta x)$;

(4) $\beta$ the probability of breakdown arrival;

(5) $\lambda C_{i}$ the probability of batch arrival.

Consider the following

$$
\begin{aligned}
& P_{n, 1}(x, \Delta x)=\left(1-\left\{\lambda+\mu_{1}(x)+\beta\right\} \Delta x\right) \\
& \times P_{n, 1}(x)+P_{n-1,1}(x) \lambda C_{1} \Delta x \\
&+P_{n-2,1}(x) \lambda C_{2} \Delta x \\
&+\cdots+P_{1,1}(x) \lambda C_{n-1} \Delta x, \\
& \frac{P_{n, 1}(x+\Delta x)-}{\Delta x}+P_{n, 1}(x) \\
&=\sum_{i=1}^{n} \lambda C_{i} P_{n-i, 1}(x) .
\end{aligned}
$$

From above we have

$$
\begin{aligned}
& \frac{d}{d x} P_{n, 1}(x, z)+\left\{\lambda+\mu_{1}(x)+\beta\right\} P_{n, 1}(x) \\
& \quad=\lambda \sum_{i=1}^{n} c_{i} P_{n-i, 1}(x) \quad n \geq 1 .
\end{aligned}
$$

Similarly we have

$$
\begin{aligned}
& \frac{d}{d x} P_{n, 2}(x, z)+\left\{\lambda+\mu_{2}(x)+\beta\right\} P_{n, 2}(x) \\
& \quad=\lambda \sum_{i=1}^{n} c_{i} P_{n-i, 2}(x) \quad n \geq 1 .
\end{aligned}
$$

For $N$ th stage, $N \geq 3$,

$$
\begin{gathered}
\frac{d}{d x} P_{n, N}(x, z)+\left\{\lambda+\mu_{N}(x)+\beta\right\} P_{n, N}(x) \\
=\lambda \sum_{i=1}^{n} c_{i} P_{n-i, N}(x) \quad n \geq 1, \\
\frac{d}{d x} R_{n}(x)+\{\lambda+\Upsilon(x)+\psi\} R_{n}(x) \\
=\lambda \sum_{i=1}^{n} c_{i} R_{n-i}(x)+\psi R_{n}(x) \quad n \geq 1, \\
\frac{d}{d x} R_{0}(x)+(\lambda+\Upsilon(x)) R_{0}(x)=\psi R_{1}(x), \\
\frac{d}{d x} V_{n}(x)+\{\lambda+\xi(x)+\psi\} V_{n}(x) \\
=\lambda \sum_{i=1}^{n} c_{i} V_{n-i}(x)+\psi V_{n+1}(x) \quad n \geq 1, \\
\frac{d}{d x} V_{0}(x)+\{\lambda+\xi(x)+\psi\} V_{0}(x)=\psi V_{0}(x), \\
\lambda Q=(1-\theta) \int_{0}^{\infty} P_{0, N}(x) \mu_{N}(x) d x \\
+\int_{0}^{\infty} R_{0}(x) \Upsilon(x) d x .
\end{gathered}
$$

The abovementioned differential equations should be solved based on the following boundary conditions:

$$
\begin{aligned}
P_{n, 1}(0)= & \lambda c_{n} Q+(1-\theta) \int_{0}^{\infty} P_{n+1, N}(x) \mu_{N}(x) d x \\
& +\int_{0}^{\infty} R_{n+1}(x) \Upsilon(x) d x \\
& +\int_{0}^{\infty} V_{n}(x) \psi(x) d x \quad n \geq 1, \\
P_{n, 2}(0)= & \int_{0}^{\infty} P_{n, 1}(x) \mu_{1}(x) d x \quad n \geq 1 .
\end{aligned}
$$


For Nth stage,

$$
\begin{gathered}
P_{n, N}(0)=\int_{0}^{\infty} P_{n, N-1}(x) \mu_{N-1}(x) d x \quad n \geq 1, \\
V_{n}(0)=\theta \int_{0}^{\infty} P_{n+1, N}(x) \mu_{N}(x) d x \quad n \geq 0 \\
R_{n+1}(0)=\sum_{j=1}^{N} \int_{0}^{\infty} P_{n, j}(x) d x \\
R_{0}(0)=0 .
\end{gathered}
$$

Note. LHS of (14) indicates that there are $n$ customers in the system and the service is about to start in stage 1 .

RHS of (14) indicates the various situations in which the system has $n$ customers and the service is about to start in stage 1.

The first term of RHS of (14) indicates that the system is in idle $(Q)$, an arrival of batch consisting of $n$ customers occurs, and 1st stage service is about to start.

The second term indicates that there are $n+1$ customers in the system including one customer whose service is completed in $N$ th stage $(N \geq 2)$ with probability $\mu_{N}(x)$ remaining $n$ customers are there in the system. 1st stage of service is about to start. Moreover the server is not going for vacation; instead stays in the system with probability $1-\theta$.

The third term indicates the repair process gets completed with probability $\Upsilon(x)$. So one customer just enters into the 1st stage service. Now there are $n$ customers in the system.

The fourth term indicates that vacation gets completed with probability $\psi(x)$. There are $n$ customers in the system. 1st stage of service is about to start. (14).

The combination of all the four terms gives the LHS of

The integral in the RHS of (15) states that, after completion of 1st stage of service, there are $n$ customers in the system to whom 2 nd stage of service is about to start which gives the LHS of (15).

Similarly (16) indicates that the Nth stage of service is about to start.

RHS of (17) explains the fact that, after completion of vacation with probability $\theta$, the server has just returned to the system consisting of $n$ customers and the 1st stage of service is about to start. This leads to the LHS of (17).

\section{Queue Size Distribution at Random Epoch}

We use supplementary variable technique to get the following comprehensive idea about the model.

Multiplying (6) to (12) by $z^{n}$ and summing over $n$ from 1 to $\infty$ yields that we can obtain

$$
\begin{aligned}
& \frac{d}{d x} P_{1}(x, z)+\left\{(\lambda-\lambda C(z))+\mu_{1}(x)+\beta\right\} P_{1}(x, z)=0 \\
& \frac{d}{d x} P_{2}(x, z)+\left\{(\lambda-\lambda C(z))+\mu_{2}(x)+\beta\right\} P_{2}(x, z)=0 .
\end{aligned}
$$

For Nth stage,

$$
\begin{aligned}
& \frac{d}{d x} P_{N}(x, z)+\left\{(\lambda-\lambda C(z))+\mu_{N}(x)+\beta\right\} P_{N}(x, z)=0 \\
& \frac{d}{d x} R(x, z)+\left\{\lambda-\lambda C(z)+\Upsilon(x)+\psi-\frac{\psi}{2}\right\} R(x, z)=0 \\
& \frac{d}{d x} V(x, z)+\left\{\lambda-\lambda C(z)+\xi(x)+\psi-\frac{\psi}{z}\right\}=0
\end{aligned}
$$

Further integration of (20)-(23) over limits 0 to $x$, in general, will result in

$$
\begin{aligned}
& P_{j}(x, z)=P_{j}(0, z) e^{\left[(\lambda-\lambda C(z)+\beta) x-\int_{0}^{\infty} \mu_{j}(t) d t\right]} j=1 \text { to } N, \\
& R(x, z)=R(0, z) e^{\left[(\lambda-\lambda C(z)+\psi-(\psi / z)) x-\int_{0}^{\infty} \Upsilon(t) d t\right]}, \\
& V(x, z)=V(0, z) e^{\left[(\lambda-\lambda C(z)+\psi-(\psi / z)) x-\int_{0}^{\infty} \xi(t) d t\right]} .
\end{aligned}
$$

Next, by multiplying the boundary conditions by suitable powers of $z^{n+1}$, taking summation over all possible values of $n$, and using the probability generating functions, we get

$$
\begin{aligned}
& z P_{1}(0, z)=(\lambda C(z)-\lambda) Q+(1-\theta) \int_{0}^{\infty} P_{N}(x, z) \mu_{N}(x) d x \\
&+\int_{0}^{\infty} R(x, z) \Upsilon(x) d x \\
&+\int_{0}^{\infty} V(x, z) \xi(x) d x, \\
& P_{2}(0, z)=\int_{0}^{\infty} P_{1}(x, z) \mu_{1}(x) d x .
\end{aligned}
$$

For Nth stage,

$$
\begin{aligned}
& P_{N}(0, z)=\int_{0}^{\infty} P_{N-1}(x, z) \mu_{N-1}(x) d x, \\
& z V(0, z)=\theta \int_{0}^{\infty} P_{N}(x, z) \mu_{N}(x) d x, \\
& R(0, z)=\alpha z \sum_{j=1}^{N} P_{j}(z) .
\end{aligned}
$$

Again integrating (24) with respect to $x$, in general, will result in

$$
P_{j}(z)=P_{j}(0, z)\left[\frac{1-\bar{K}_{j}(\lambda-\lambda C(z)+\beta)}{\lambda-\lambda C(z)+\beta}\right] j=1 \text { to } N,
$$

$$
\begin{aligned}
& V(z)=V(0, z)\left[\frac{1-\bar{M}(\lambda-\lambda C(z)+\psi-(\psi / z))}{\lambda-\lambda C(z)+\psi-(\psi / z)}\right], \\
& R(z)=R(0, z)\left[\frac{1-\bar{G}(\lambda-\lambda C(z)+\psi-(\psi / z))}{\lambda-\lambda C(z)+\psi-(\psi / z)}\right],
\end{aligned}
$$


where

$$
\begin{gathered}
\bar{K}_{j}(\lambda-\lambda C(z)+\beta)=\int_{0}^{\infty} e^{-(\lambda-\lambda C(z)+\beta) x} d K_{j}(x), \\
\bar{G}\left(\lambda-\lambda C(z)+\psi-\frac{\psi}{z}\right)=\int_{0}^{\infty} e^{-(\lambda-\lambda C(z)+\psi-(\psi / z)) x} d G(x), \\
\bar{M}\left(\lambda-\lambda C(z)+\psi-\frac{\psi}{z}\right)=\int_{0}^{\infty} e^{-(\lambda-\lambda C(z)+\psi-(\psi / z)) x} d M(x)
\end{gathered}
$$

are the Laplace-Stieltjes transform of the $j$ th stage repair time and vacation time, respectively.

By multiplying the RHS of $(24)$ by $\mu_{j}(x), \Upsilon(x)$, and $\xi(x)$, respectively, and integrating with respect to $x$, we can obtain

$$
\begin{array}{r}
\int_{0}^{\infty} P_{j}(x, z) \mu_{j}(x) d x=P_{j}(0, z) \bar{K}_{j}(\lambda-\lambda C(z)+\beta) \\
j=1 \text { to } N \\
\int_{0}^{\infty} R(x, z) \Upsilon(x) d x=R(0, z) \bar{G}\left(\lambda-\lambda C(z)+\psi-\frac{\psi}{z}\right), \\
\int_{0}^{\infty} V(x, z) \xi(x) d x=V(0, z) \bar{M}\left(\lambda-\lambda C(z)+\psi-\frac{\psi}{z}\right) .
\end{array}
$$

Let us take

$$
\lambda-\lambda C(z)+\beta=h_{1} ; \quad \lambda-\lambda C(z)+\psi-\frac{\psi}{z}=h_{2} .
$$

By using (33) in (25)-(27), we can obtain

$$
\begin{aligned}
z P_{1}(0, z)= & (\lambda C(z)-\lambda) Q+(1-\theta) \bar{K}_{N}\left(h_{1}\right) P_{N}(0, z) \\
+ & R(0, z) \bar{G}\left(h_{2}\right)+z V(0, z) \bar{M}\left(h_{2}\right), \\
& P_{2}(0, z)=P_{1}(0, z) \bar{K}_{1}\left(h_{1}\right) .
\end{aligned}
$$

For Nth stage

$$
\begin{gathered}
P_{N-1}(0, z)=P_{N-1}(0, z) \bar{K}_{N-1}\left(h_{1}\right), \\
V(0, z)=\theta P_{N}(0, z) \prod_{j=1}^{N} \bar{K}_{j}\left(h_{1}\right) .
\end{gathered}
$$

By employing (36b) in (37), we can get

$$
z V(0, z)=\theta P_{1}(0, z) \prod_{j=1}^{N} \bar{K}_{j}\left(h_{1}\right) .
$$

Again, by using (28) in (29), we get

$$
R(0, z)=\frac{\beta z}{h_{1}}\left[\sum_{j=1}^{N} P_{j}(0, z)\left(1-\bar{K}_{j}(m)\right)\right] .
$$

Now by employing (36b), (38), and (39) in (35), we can solve for $P_{1}(0, z)$ :

$$
P_{1}(0, z)=\frac{h_{1}(\lambda C(z)-\lambda) Q}{D(z)}
$$

$D(z)$

$$
\begin{aligned}
= & h_{1}\left[z-(1-\theta) \prod_{j=1}^{N} \bar{K}_{j}\left(h_{1}\right)-\theta \prod_{j=1}^{N} \bar{K}_{j}\left(h_{1}\right) \bar{M}(k)\right] \\
& -\beta z \bar{G}(k)\left\{1-\prod_{j=1}^{N} \bar{K}_{j}\left(h_{1}\right)\right\},
\end{aligned}
$$

$$
\begin{gathered}
P_{2}(0, z)=\frac{h_{1}(\lambda C(z)-\lambda) \bar{K}_{1}\left(h_{1}\right) Q}{D(z)}, \\
P_{N}(0, z)=\frac{h_{1}(\lambda C(z)-\lambda) \bar{K}_{N-1}\left(h_{1}\right) Q}{D(z)}, \\
V(0, z)=\frac{\theta h_{1}(\lambda C(z)-\lambda) \prod_{j=1}^{N} \bar{K}_{j}\left(h_{1}\right) Q}{D(z)} .
\end{gathered}
$$

By substituting (40), (42), (43), and (44) in (29)-(31), we can obtain

$$
\begin{aligned}
& P_{1}(z)=\frac{(\lambda C(z)-\lambda)\left[1-\bar{K}_{1}\left(h_{1}\right)\right] Q}{D(z)}, \\
& P_{2}(z)=\frac{(\lambda C(z)-\lambda) \bar{K}_{1}\left(h_{1}\right)\left[1-\bar{K}_{2}\left(h_{1}\right)\right] Q}{D(z)} .
\end{aligned}
$$

For $N$ th stage

$$
\begin{aligned}
& P_{N}(z)=\frac{(\lambda C(z)-\lambda) \bar{K}_{N-1}\left(h_{1}\right)\left[1-\bar{K}_{N}\left(h_{1}\right)\right] Q}{D(z)}, \\
& R(z)=\frac{\beta z(\lambda C(z)-\lambda)\left[1-\prod_{j=1}^{N} \bar{K}_{j}\left(h_{1}\right)\right] Q}{D(z)}\left[\frac{1-\bar{G}\left(h_{2}\right)}{h_{2}}\right],
\end{aligned}
$$

$$
\begin{aligned}
V(z)= & \frac{\theta h_{1}(\lambda C(z)-\lambda)\left[1-\prod_{j=1}^{N} \bar{K}_{j}\left(h_{1}\right)\right] Q}{D(z)} \\
& \times\left[\frac{1-\bar{M}\left(h_{2}\right)}{h_{2}}\right] .
\end{aligned}
$$

Let $P_{\mathrm{Q}}(z)$ denote the probability generating function of the queue size irrespective of the state of the system:

$$
P_{\mathrm{Q}}(z)=P_{1}(z)+P_{2}(z)+P_{3}(z)+R(z)+V(z)=\frac{N(z)}{D(z)} .
$$

To determine the probability of idle time $Q$, we use the normalizing condition

$$
P_{\mathrm{Q}}(1)+\mathrm{Q}=1
$$


Again, as (49) is indeterminate of the form $0 / 0$ at $z=1$, L'Hopital's rule is employed in (49) to obtain

$$
\begin{aligned}
P_{1}(1) & =\frac{\lambda E(I)\left(1-\bar{K}_{1}(\beta)\right) Q}{D R}, \\
P_{2}(1) & =\frac{\lambda E(I) \bar{K}_{1}(\beta)\left(1-\bar{K}_{2}(\beta)\right) Q}{D R}, \\
P_{N}(1) & =\frac{\lambda E(I) Q \prod_{j=1}^{N} \bar{K}_{j-1}(\beta)\left(1-\bar{K}_{j}(\beta)\right)}{D R} .
\end{aligned}
$$

Equations (51), (52) and (53) indicate the steady-state probability of the server in stages 1,2 , and $N$, respectively.

It must be noted that

$$
\begin{aligned}
& R(1)=\frac{\beta \lambda E(I) E(R)\left[1-\prod_{j=1}^{N} \bar{K}_{j}(\beta)\right] Q}{D R}, \\
& V(1)=\frac{\theta \beta \lambda E(I) E(V) \prod_{j=1}^{N} \bar{K}_{j}(\beta) Q}{D R},
\end{aligned}
$$

where $D R=-(\lambda E(I)+\beta(\lambda E(I)-\psi) E(R))+[\beta+\lambda E(I)+$ $\beta(\lambda E(I)-\psi) E(R)-\theta \beta(\lambda E(I)-\psi) E(v)] \prod_{j=1}^{N} \bar{K}_{j}(\beta)$.

Thus the normalization condition yields

$$
\begin{aligned}
& Q=1 \\
& -((\lambda E(I)[\{1+\beta E(R)\} \\
& \left.\left.-\{1+\beta E(R)-\theta \beta E(V)\} \prod_{j=1}^{N} \bar{K}_{j}(\beta)\right]\right) \\
& \times\left(\beta \psi E(R)\left\{1-\prod_{j=1}^{N} \bar{K}_{j}(\beta)\right\}\right. \\
& \left.\left.+\theta \beta \psi \prod_{j=1}^{N} \bar{K}_{j}(\beta)\right)^{-1}\right)
\end{aligned}
$$

and the utilization factor $\rho=1-Q$ is

$$
\begin{gathered}
\rho=(\lambda E(I)[\{1+\beta E(R)\} \\
-\{1+\beta E(R)-\theta \beta E(V)\} \\
\left.\left.\times \prod_{j=1}^{N} \bar{K}_{j}(\beta)\right]\right) \\
\times\left(\beta \psi E(R)\left\{1-\prod_{j=1}^{N} \bar{K}_{j}(\beta)\right\}\right. \\
\left.+\theta \beta \psi \prod_{j=1}^{N} \bar{K}_{j}(\beta)\right)^{-1}<1 .
\end{gathered}
$$

\section{Average Queue Size and Average Waiting Time}

As $L_{q}=\left.(d / d z) P_{q}(z)\right|_{z=1}$, with the mean queue size is of the $0 / 0$ form, the L'Hopital's rule is applied twice to obtain

$$
L_{q}=\lim _{z \rightarrow 1} \frac{D^{\prime}(z) N^{\prime \prime}(z)-N^{\prime}(z) D^{\prime \prime}(z)}{2\left(D^{\prime}(z)\right)^{2}},
$$

where the primes and double primes denote the first and second derivatives, respectively:

$$
\begin{aligned}
& N^{\prime}(1)=Q[\lambda E(I)\{1+\beta E(R)\} \\
& -\lambda E(I)\{1+\beta E(R)-\theta \beta E(V)\} \\
& \left.\times \prod_{j=1}^{N} \bar{K}_{j}(\beta)\right] \\
& N^{\prime \prime}(1)=Q\left[\lambda E\left(\frac{I}{I-1}\right)\right. \\
& \times\left\{\left\{\left[1-\prod_{j=1}^{N} \bar{K}_{j}(\beta)\right]\right.\right. \\
& +\beta\left[1-\prod_{j=1}^{N} \bar{K}_{j}(\beta)\right] E(R) \\
& \left.+\theta \beta \prod_{j=1}^{N} \bar{K}_{j}(\beta) E(V)\right\} \\
& +\beta(\lambda E(I)-\psi) E\left(R^{2}\right)
\end{aligned}
$$




$$
\begin{aligned}
& \times\left[1-\prod_{j=1}^{N} \bar{K}_{j}(\beta)\right] \\
& +(\lambda E(I)-\psi) E\left(R^{2}\right) \\
& \left.\left.\times \prod_{j=1}^{N} \bar{K}_{j}(\beta)\right\}\right] \text {, } \\
& D^{\prime}(1)=-\{\lambda E(I)+\beta(\lambda E(I)-\psi) E(R)\} \\
& +\{\lambda E(I)+\beta(\lambda E(I)-\psi) E(R) \\
& -\theta \beta(\lambda E(I)-\psi) E(V)+\beta\} \\
& \times \prod_{j=1}^{N} \bar{K}_{j}(\beta), \\
& D^{\prime \prime}(1)=-\lambda E\left(\frac{I}{I-1}\right) \\
& \times\left[(1+\beta E(R))-\prod_{j=1}^{N} \bar{K}_{j}(\beta)\right. \\
& \times\{1-\beta E(R)+\theta \beta E(V)\}] \\
& -2 \beta \psi E(R)\left\{1-\prod_{j=1}^{N} \bar{K}_{j}(\beta)\right\} \\
& +\beta(\lambda E(I)-\psi)^{2} E\left(R^{2}\right) \\
& \times\left(1-\prod_{j=1}^{N} \bar{K}_{j}(\beta)\right) \\
& -\theta \beta\left\{2 \psi E(V)-(\lambda E(I)-\psi)^{2} E\left(V^{2}\right)\right\} \\
& \times \prod_{j=1}^{N} \bar{K}_{j}(\beta) .
\end{aligned}
$$

By utilizing (58) in (57), we can obtain the mean length of the queue size $L_{q}$ while the mean waiting time of the queue $W_{q}$ can be derived by using $W_{q}=L_{q} / \lambda$. Furthermore, we can also determine $L=L_{q}+\rho$, the mean queue size of the system, and $W=L / \lambda$, the mean waiting time of the system.

\section{Particular Cases}

Case 1 (no server vacations). In this case, the server has no vacation. Hence, $V(z)=0$. Thus $\theta=0$ in (45)-(47). Our model reduces to multistage batch arrivals with reneging during breakdowns, and we have

$$
\begin{aligned}
& P_{1}(z)=\frac{(\lambda C(z)-\lambda)\left[1-\bar{K}_{1}\left(h_{1}\right)\right] Q}{D(z)}, \\
& P_{2}(z)=\frac{(\lambda C(z)-\lambda) \bar{K}_{1}\left(h_{1}\right)\left[1-\bar{K}_{2}\left(h_{1}\right)\right] Q}{D(z)} .
\end{aligned}
$$

For Nth stage,

$$
\begin{aligned}
& P_{N}(z)=\frac{(\lambda C(z)-\lambda) \bar{K}_{N-1}\left(h_{1}\right)\left[1-\bar{K}_{N}\left(h_{1}\right)\right] Q}{D(z)}, \\
& R(z)=\frac{\beta z(\lambda C(z)-\lambda)\left[1-\prod_{j=1}^{N} \bar{K}_{j}\left(h_{1}\right)\right] Q}{D(z)}\left[\frac{1-\bar{G}\left(h_{2}\right)}{h_{2}}\right], \\
& D(z)=h_{1}\left[z-\prod_{j=1}^{N} \bar{K}_{j}\left(h_{1}\right)\right]-\beta z \bar{G}\left(h_{2}\right) \prod_{j=1}^{N} \bar{K}_{j}\left(h_{1}\right) .
\end{aligned}
$$

The probability of idle time is

$$
Q=1-\frac{\lambda E(I)\left[\{1+\beta E(R)\}-\{1+\beta E(R)\} \prod_{j=1}^{N} \bar{K}_{j}(\beta)\right]}{\beta \psi E(R)\left\{1-\prod_{j=1}^{N} \bar{K}_{j}(\beta)\right\}}
$$

and the traffic intensity $\rho$ is

$$
\rho=\frac{\lambda E(I)\left[\{1+\beta E(R)\}-\{1+\beta E(R)\} \prod_{j=1}^{N} \bar{K}_{j}(\beta)\right]}{\beta \psi E(R)\left\{1-\prod_{j=1}^{N} \bar{K}_{j}(\beta)\right\}} .
$$

Furthermore by considering $\theta=0$ in (58) we can obtain the mean queue size $L_{q}$ and mean waiting time $W_{q}$.

Case 2 (exponential service time and exponential vacation time). In this case, we assume that the service time for the multistage of service with service rate $\mu_{j}>0, j=1$ to $N$, is exponentially distributed. Furthermore, the repair time and vacation time are exponentially distributed with a repair rate $\Upsilon>0$ and vacation time $\xi>0$.

Thus,

$$
\begin{gathered}
\bar{K}_{1}\left(h_{1}\right)=\frac{\mu_{j}}{\mu_{j}+h_{1}} ; \quad j=1 \text { to } N, \\
\bar{G}\left(h_{2}\right)=\frac{\Upsilon}{\Upsilon+h_{2}} ; \quad \bar{M}\left(h_{2}\right)=\frac{\xi}{\xi+h_{2}}, \\
E(R)=\frac{1}{\Upsilon} ; \quad E\left(R^{2}\right)=\frac{2}{\Upsilon^{2}}, \\
E(V)=\frac{1}{\xi} ; \quad E\left(V^{2}\right)=\frac{2}{\xi^{2}},
\end{gathered}
$$

where $h_{1}=\lambda-\lambda C(z)+\beta ; \quad h_{2}=\lambda-\lambda C(z)+\psi-(\psi / z)$. 
By utilizing the abovementioned relations in (44)-(46), we can obtain

$$
\begin{aligned}
& P_{1}(z)=\frac{(\lambda C(z)-\lambda)\left[1-\left(\mu_{1} /\left(\mu_{1}+h_{1}\right)\right)\right] Q}{D(z)}, \\
& P_{2}(z)=\frac{(\lambda C(z)-\lambda)\left(\mu_{1} /\left(\mu_{1}+h_{1}\right)\right)\left[1-\left(\mu_{2} /\left(\mu_{2}+h_{1}\right)\right)\right] Q}{D(z)} .
\end{aligned}
$$

For Nth stage,

$$
\begin{aligned}
P_{N}(z)= & \left((\lambda C(z)-\lambda) \frac{\mu_{1}}{\mu_{N-1}+h_{1}}\left[1-\frac{\mu_{N}}{\mu_{N}+h_{1}}\right] Q\right) \\
& \times(D(z))^{-1}, \\
R(z)=( & (\lambda C(z)-\lambda)\left[1-\frac{\prod_{j=1}^{N} \mu_{j}}{\prod_{j=1}^{N}\left(\mu_{j}+h_{1}\right)}\right] \\
& \left.\times \frac{1}{\left(\Upsilon+h_{2}\right)} Q\right) \times(D(z))^{-1}, \\
V(z)=( & \left.\theta h_{1}(\lambda C(z)-\lambda) \frac{\prod_{j=1}^{N} \mu_{j}}{\prod_{j=1}^{N}\left(\mu_{j}+h_{1}\right)\left(\psi+h_{2}\right)} Q\right) \\
& \times(D(z))^{-1},
\end{aligned}
$$

where

$$
\begin{aligned}
D(z)= & (\lambda-\lambda C(z)+\beta) \\
& \times\left[z-\left\{(1-\theta)+\theta \frac{\xi}{\xi+h_{2}}\right\} \frac{\prod_{j=1}^{N} \mu_{j}}{\prod_{j=1}^{N}\left(\mu_{j}+h_{1}\right)}\right] \\
& -\beta z\left\{1-\frac{\prod_{j=1}^{N} \mu_{j}}{\prod_{j=1}^{N}\left(\mu_{j}+h_{1}\right)}\right\} \frac{\Upsilon}{\left(\Upsilon+h_{2}\right)} .
\end{aligned}
$$

Therefore, the probability that the server is providing service in the first, second, and $N$ th stages at a random point of time can be given as presented in (67), (68), and (69), respectively. Consider the following:

$$
\begin{aligned}
P_{1}(1)= & \left(\lambda E(I)\left[1-\frac{\mu_{1}}{\mu_{1}+\beta}\right] Q\right) \\
& \times-\left[\left(\lambda E(I)+\frac{\beta(\lambda E(I)-\psi)}{\Upsilon}\right)\right.
\end{aligned}
$$

$$
+[\beta+\lambda E(I)+\beta(\lambda E(I)-\psi)
$$$$
\left.\left.\times\left\{\frac{1}{\Upsilon}-\frac{\theta}{\xi}\right\}\right] \times \frac{\prod_{j=1}^{N} \mu_{j}}{\prod_{j=1}^{N}\left(\mu_{j}+\beta\right)}\right]^{-1},
$$

$$
P_{2}(1)=\left(\lambda E(I) \frac{\mu_{1}}{\mu_{1}+\beta}\left[1-\frac{\mu_{2}}{\mu_{1}+\beta}\right] Q\right)
$$$$
x-\left[\left(\lambda E(I)+\frac{\beta(\lambda E(I)-\psi)}{\Upsilon}\right)\right.
$$

$$
+[\beta+\lambda E(I)+\beta(\lambda E(I)-\psi)
$$

$$
\left.\left.\times\left\{\frac{1}{\Upsilon}-\frac{\theta}{\psi}\right\}\right] \times \frac{\prod_{j=1}^{N} \mu_{j}}{\prod_{j=1}^{N}\left(\mu_{j}+\beta\right)}\right]^{-1},
$$

$$
\begin{aligned}
P_{N}(1)=\left(\lambda E(I) \frac{\mu_{N-1}}{\mu_{1}+\beta}\left[1-\frac{\mu_{N}}{\mu_{N}+\beta}\right] Q\right) \\
\times-\left[\left(\lambda E(I)+\frac{\beta(\lambda E(I)-\psi)}{\Upsilon}\right)\right. \\
+[\beta+\lambda E(I)+\beta(\lambda E(I)-\psi) \\
\left.\left.\quad \times\left\{\frac{1}{\Upsilon}-\frac{\theta}{\xi}\right\}\right] \times \frac{\prod_{j=1}^{N} \mu_{j}}{\prod_{j=1}^{N}\left(\mu_{j}+\beta\right)}\right]^{-1} .
\end{aligned}
$$

Furthermore, the probability that server is under repairs at random point of time is

$$
R(1)=\left(\left[\frac{\beta E(I)}{\Upsilon}\right]\left[1-\frac{\prod_{j=1}^{N} \mu_{j}}{\prod_{j=1}^{N}\left(\mu_{j}+\beta\right)}\right] Q\right)
$$

$$
\begin{aligned}
\times-\left[\left(\lambda E(I)+\frac{\beta(\lambda E(I)-\psi)}{\Upsilon}\right)\right. \\
+[\beta+\lambda E(I)+\beta(\lambda E(I)-\psi) \\
\left.\left.\times\left\{\frac{1}{\Upsilon}-\frac{\theta}{\xi}\right\}\right] \times \frac{\prod_{j=1}^{N} \mu_{j}}{\prod_{j=1}^{N}\left(\mu_{j}+\beta\right)}\right]^{-1} .
\end{aligned}
$$


The probability that server is on vacation at random point of time is given by

$$
\begin{aligned}
V(1)= & \left(\theta \beta\left[\frac{\lambda E(I)}{\xi}\right] \frac{\prod_{j=1}^{N} \mu_{j}}{\prod_{j=1}^{N}\left(\mu_{j}+\beta\right)} Q\right) \\
\times- & {\left[\left(\lambda E(I)+\frac{\beta(\lambda E(I)-\psi)}{\Upsilon}\right)\right.} \\
& +[\beta+\lambda E(I)+\beta(\lambda E(I)-\psi) \\
& \left.\left.\times\left\{\frac{1}{\Upsilon}-\frac{\theta}{\xi}\right\}\right] \times \frac{\prod_{j=1}^{N} \mu_{j}}{\prod_{j=1}^{N}\left(\mu_{j}+\beta\right)}\right]^{-1} .
\end{aligned}
$$

The probability that the server is idle but available in the system is given by

$$
\begin{aligned}
Q=1 & -\lambda E(I) \\
\times & {\left[\left\{1+\frac{\beta}{\Upsilon}\right\}-\left\{1+\frac{\beta}{\Upsilon}-\frac{\theta \beta}{\psi}\right\} \frac{\prod_{j=1}^{N} \mu_{j}}{\prod_{j=1}^{N}\left(\mu_{j}+\beta\right)}\right] } \\
\times & \left(\frac{\beta \psi}{\Upsilon}\left\{1-\frac{\prod_{j=1}^{N} \mu_{j}}{\prod_{j=1}^{N}\left(\mu_{j}+\beta\right)}\right\}\right. \\
& \left.+\theta \beta \psi \frac{\prod_{j=1}^{N} \mu_{j}}{\prod_{j=1}^{N}\left(\mu_{j}+\beta\right)}\right)^{-1} .
\end{aligned}
$$

Similarly the mean queue size and mean waiting time can be derived by determining $N^{\prime}(1), N^{\prime \prime}(1), D^{\prime}(1)$, and $D^{\prime \prime}(1)$ and utilizing them in (57). Consider the following:

$$
\begin{aligned}
N^{\prime}(1)=Q[ & \lambda E(I)\left\{1+\frac{\beta}{\Upsilon}\right\}-\lambda E(I)\left\{1+\frac{\beta}{\Upsilon}-\frac{\theta \beta}{\Upsilon}\right\} \\
& \left.\times \frac{\prod_{j=1}^{N} \mu_{j}}{\prod_{j=1}^{N}\left(\mu_{j}+\beta\right)}\right],
\end{aligned}
$$

$N^{\prime \prime}(1)$

$$
\begin{aligned}
=Q[\lambda & \left(\frac{I}{I-1}\right) \\
& \times\left\{\left(1+\frac{\beta}{\Upsilon}\right)\right. \\
& \times\left(1-\frac{\prod_{j=1}^{N} \mu_{j}}{\prod_{j=1}^{N}\left(\mu_{j}+\beta\right)}\right) \\
& \left.+\frac{\theta \beta}{\Upsilon} \frac{\prod_{j=1}^{N} \mu_{j}}{\prod_{j=1}^{N}\left(\mu_{j}+\beta\right)}\right\}
\end{aligned}
$$

$$
-2 \lambda E(I)
$$

$$
\begin{gathered}
\times\left\{\frac{\beta(\lambda E(I)-\psi)}{\Upsilon^{2}}\right. \\
\quad \times\left(1-\frac{\prod_{j=1}^{N} \mu_{j}}{\prod_{j=1}^{N}\left(\mu_{j}+\beta\right)}\right) \\
+\frac{\theta(\lambda E(I)-\psi)}{\xi^{2}} \\
\left.\left.\quad \times \frac{\prod_{j=1}^{N} \mu_{j}}{\prod_{j=1}^{N}\left(\mu_{j}+\beta\right)}\right\}\right]
\end{gathered}
$$

$D^{\prime}(1)$

$$
\begin{aligned}
& =-\left[\lambda E(I)+\frac{\beta(\lambda E(I)-\psi)}{\Upsilon}\right] \\
& +\left\{\beta+\lambda E(I)+\frac{\beta(\lambda E(I)-\psi)}{\Upsilon}\right. \\
& \left.-\frac{\theta \beta(\lambda E(I)-\psi)}{\xi}\right\} \\
& \times \frac{\prod_{j=1}^{N} \mu_{j}}{\prod_{j=1}^{N}\left(\mu_{j}+\beta\right)}, \\
& D^{\prime \prime}(1)=-\lambda E\left(\frac{I}{I-1}\right) \\
& \times\left\{\left(1+\frac{\beta}{\Upsilon}\right) \frac{\prod_{j=1}^{N} \mu_{j}}{\prod_{j=1}^{N}\left(\mu_{j}+\beta\right)}\left(1-\frac{\beta}{\Upsilon}+\frac{\theta \beta}{\xi}\right)\right\} \\
& -2\left[\frac{\beta \psi}{\Upsilon}+\frac{(\lambda E(I)-\psi)^{2}}{\Upsilon^{2}}\right] \\
& \times\left[1-\frac{\prod_{j=1}^{N} \mu_{j}}{\prod_{j=1}^{N}\left(\mu_{j}+\beta\right)}\right] \\
& -2 \theta \beta\left[\frac{\psi}{\xi}+\frac{(\lambda E(I)-\psi)^{2}}{\xi^{2}}\right] \\
& \times \frac{\prod_{j=1}^{N} \mu_{j}}{\prod_{j=1}^{N}\left(\mu_{j}+\beta\right)} \\
& -\left\{\lambda E(I)+\frac{\beta(\lambda E(I)-\psi)}{\Upsilon}\right. \\
& \left.-\frac{\theta \beta(\lambda E(I)-\psi)}{\xi}+\beta\right\} \\
& \times\left\{\prod_{j=1}^{N} \mu_{j} \times(1\right.
\end{aligned}
$$




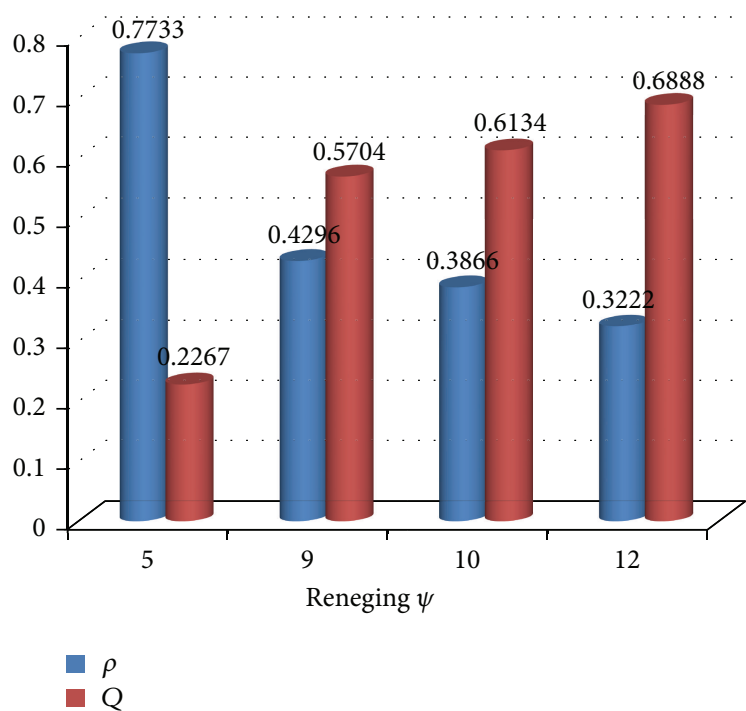

FIGURE 5: Effect of reneging $\psi=5,9,10$, and 12 and breakdown at $\beta=1$ on the proportion of idle time $Q$ and utilization factor $\rho$. From the figure, it is clear that as the idle time of the server $Q$ increases, it leads to a decrease in utilization factor $\rho$.

$$
\begin{gathered}
\times\left[\left(\mu_{1}+\beta\right)^{2} \prod_{j=2}^{N}\left(\mu_{j}+\beta\right)\right. \\
+\left(\mu_{1}+\beta\right)\left(\mu_{2}+\beta\right)^{2} \\
\times \prod_{j=3}^{N}\left(\mu_{j}+\beta\right)+\cdots \\
+\left[\left(\mu_{1}+\beta\right)\left(\mu_{2}+\beta\right) \cdots\right. \\
\left.\left.\left.\left.\quad \times\left(\mu_{N}+\beta\right)^{2}\right]\right]^{-1}\right)\right\} .
\end{gathered}
$$

\section{Numerical Illustration}

To compute the numerical results we consider the number of stages to be $N=3$; we assume service time, vacation time, and repair time to be exponentially distributed. Furthermore, let us assume that the arrivals come one after the other; that is, $E(I)=1$ and $E(I / I-1)=0$.

To monitor the effect of reneging $\psi$ and breakdown $\beta$ on the behavior of the queueing model, let us consider $\lambda=2$, $\theta=0.5, \lambda=2, \mu_{1}=3, \mu_{2}=4, \mu_{3}=5, \Upsilon=8$, and $\xi=6$, with the values of $\psi$ being $5,9,10$, and 12 and $\beta$ varying between 1 and 4 (Table 1).

From the values presented in Table 1, it can be concluded that, with the increase in the value of the parameter of customer's impatience reneging $\psi$, for varying values of the breakdown parameter $\beta$, the utilization factor $\rho$ decreases, whereas the probability of the server idle time $Q$ increases.

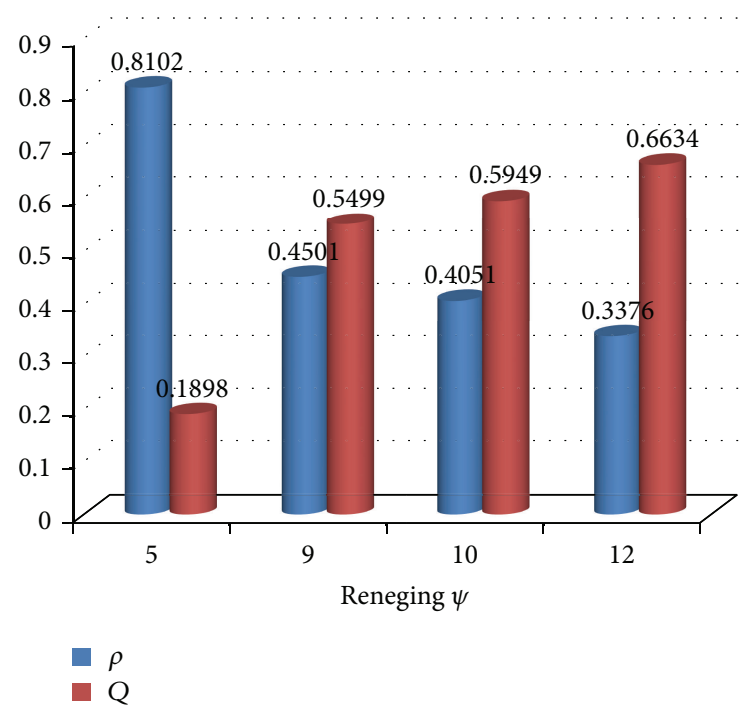

FIGURE 6: Effect of reneging $\psi=5,9,10$, and 12 and breakdown at $\beta=2$ on the proportion of idle time $Q$ and utilization factor. The graph indicates that as the idle time of the server $Q$ increases, the utilization factor $\rho$ decreases.

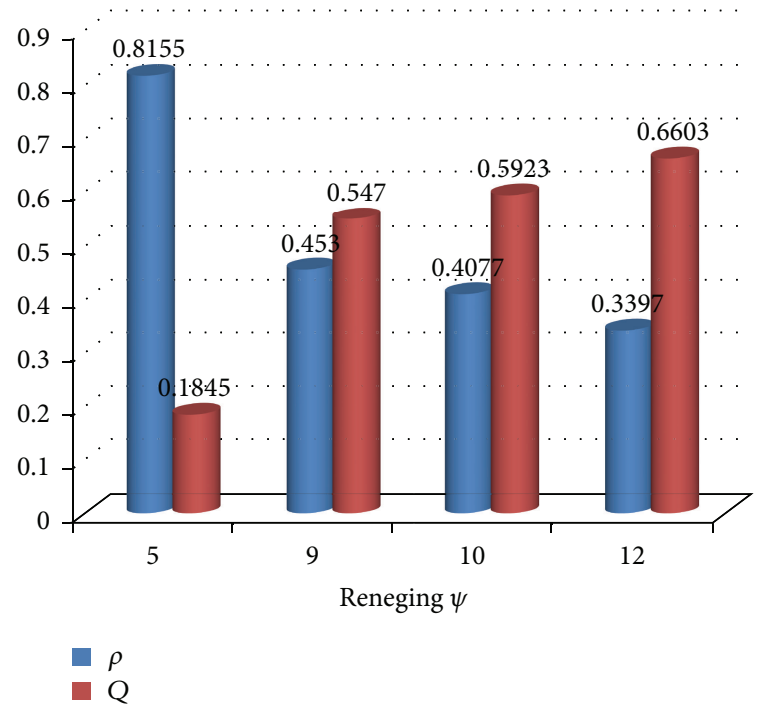

Figure 7: Effect of reneging $\psi=5,9,10$, and 12 and breakdown at $\beta=3$ on the proportion of idle time $Q$ and utilization factor $\rho$. The figure explains that as the idle time of the server $Q$ increases, the utilization factor $\rho$ decreases.

Figures $5,6,7$, and 8 clearly show that owing to the breakdown of the system and reneging, the proportion of the idle time of the server increases and the utilization factor decreases. Furthermore, Figures 9, 10, 11, and 12 demonstrate the effect of reneging and breakdown on the mean queue size $L_{q}$ and the mean waiting time $W_{q}$ and evidently indicate that, as the breakdown occurs as well as customers reneging from the queue, the average length of the queue decreases and the average waiting time decreases. 
TABLE 1: Effect of reneging and breakdown.

\begin{tabular}{|c|c|c|c|c|c|c|c|}
\hline$\psi$ & $\beta$ & $\rho$ & Q & $L_{q}$ & $L$ & $W_{q}$ & $W$ \\
\hline \multirow{4}{*}{5} & 1 & 0.7733 & 0.2267 & 4.3678 & 5.141 & 2.184 & 2.571 \\
\hline & 2 & 0.8102 & 0.1898 & 3.6436 & 4.454 & 1.822 & 2.227 \\
\hline & 3 & 0.8155 & 0.1845 & 3.0592 & 3.875 & 1.530 & 1.937 \\
\hline & 4 & 0.8233 & 0.1767 & 2.1651 & 2.988 & 1.083 & 1.494 \\
\hline \multirow{4}{*}{9} & 1 & 0.4296 & 0.5704 & 6.5243 & 6.954 & 3.262 & 3.477 \\
\hline & 2 & 0.4501 & 0.5499 & 5.5682 & 6.018 & 2.784 & 3.009 \\
\hline & 3 & 0.4530 & 0.5470 & 4.8194 & 5.272 & 2.410 & 2.636 \\
\hline & 4 & 0.4571 & 0.5429 & 4.1254 & 4.583 & 2.063 & 2.291 \\
\hline \multirow{4}{*}{10} & 1 & 0.3866 & 0.6134 & 5.8164 & 6.203 & 2.908 & 3.102 \\
\hline & 2 & 0.4051 & 0.5949 & 4.5921 & 4.997 & 2.296 & 2.499 \\
\hline & 3 & 0.4077 & 0.5923 & 3.2457 & 3.653 & 1.623 & 1.827 \\
\hline & 4 & 0.4080 & 0.5920 & 2.1352 & 2.543 & 1.068 & 1.272 \\
\hline \multirow{4}{*}{12} & 1 & 0.3222 & 0.6888 & 4.5824 & 4.905 & 2.291 & 2.452 \\
\hline & 2 & 0.3376 & 0.6634 & 4.0261 & 4.364 & 2.013 & 2.182 \\
\hline & 3 & 0.3397 & 0.6603 & 3.5024 & 3.842 & 1.751 & 1.921 \\
\hline & 4 & 0.3398 & 0.6602 & 2.9254 & 3.265 & 1.463 & 1.633 \\
\hline
\end{tabular}

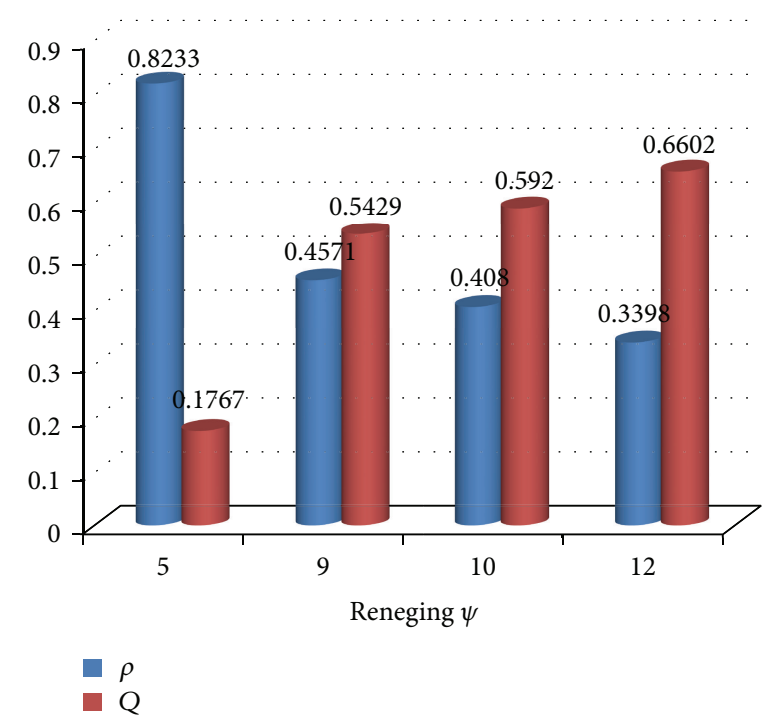

FigURE 8: Effect of reneging $\psi=5,9,10$, and 12 and breakdown at $\beta=4$ on the proportion of idle time $Q$ and utilization factor $\rho$. The figure gives the fact that as idle time of the server $Q$ increases, it leads to a decrease in utilization factor $\rho$.

\section{Conclusion}

The present study clearly analyzed the multistage batch arrival queue with reneging during vacation and breakdown periods. The steady-state solutions are obtained by using supplementary variable technique and the mean queue length and mean waiting time are calculated. Furthermore, some particular cases are also discussed and numerical illustrations are presented. It can be concluded that, with the increase in the value of the parameter of customer's impatience reneging $\psi$ for varying values of the breakdown parameter $\beta$, the

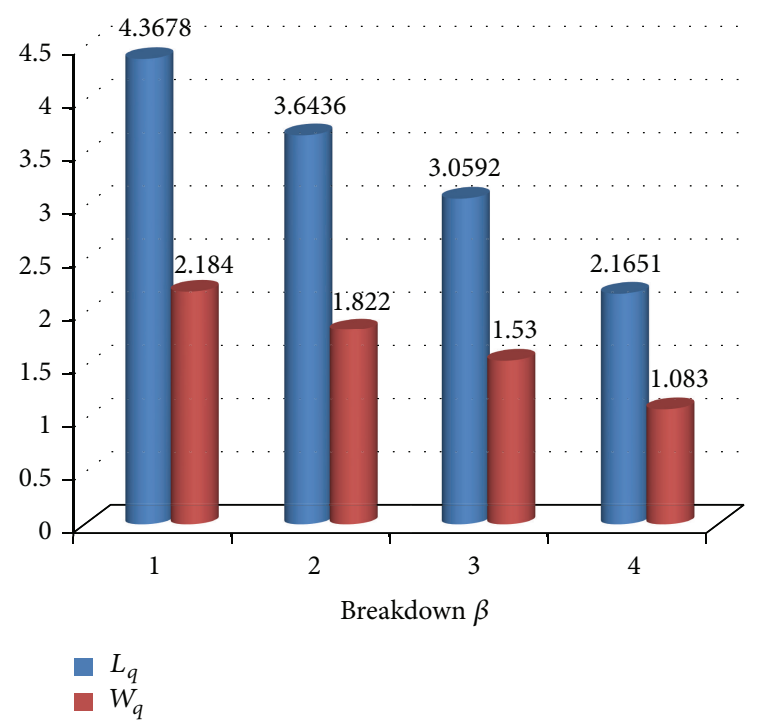

Figure 9: Effect of $\psi=5$ and $\beta=1,2,3$, and 4 on the mean queue size $L_{q}$ and mean waiting time $W_{q}$. The figure explains that the average length of the queue $L_{q}$ decreases owing to customers reneging from the queue, so the average waiting time $W_{q}$ also decreases.

utilization factor $\rho$ decreases, whereas the probability of the server idle time $Q$ increases. In this model, due to reneging and breadown the mean queue size $L_{q}$ and the mean waiting time $W_{q}$ decteases and the average waiting time decreases. For the future study compulsory vacation can be included in the present model and the effect of reneging during breakdown can be obtained. The model presented in this study can be utilized for communication networks and largescale manufacturing industries. 


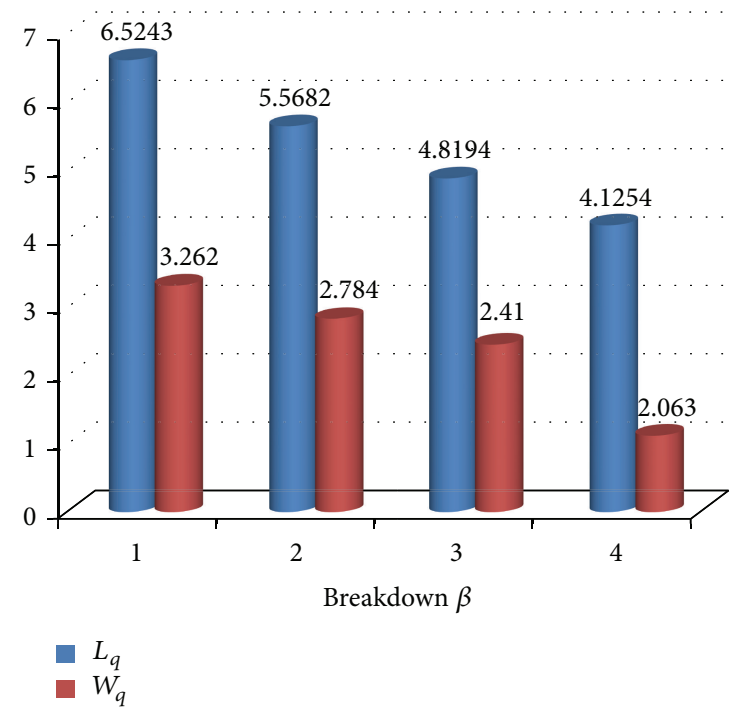

Figure 10: Effect of $\psi=9$ and $\beta=1,2,3$, and 4 on the mean queue size $L_{q}$ and mean waiting time $W_{q}$. The graph indicates that as the average length of the queue $L_{q}$ decreases, owing to customers reneging from the queue, the average waiting time $W_{q}$ also decreases.

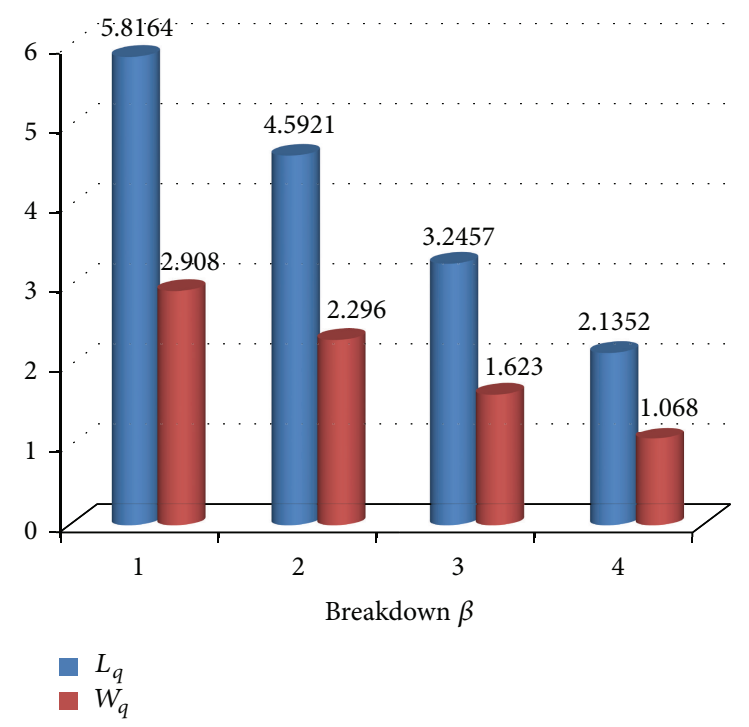

FIgURE 11: Effect of $\psi=10$ and $\beta=1,2,3$, and 4 on the mean queue size $L_{q}$ and mean waiting time $W_{q}$. The figure gives the fact that the average length of the queue $L_{q}$ decreases, owing to customers reneging from the queue, which leads to decrease in average waiting time $W_{q}$.

\section{Conflict of Interests}

The authors declare that there is no conflict of interests regarding the publication of this paper.

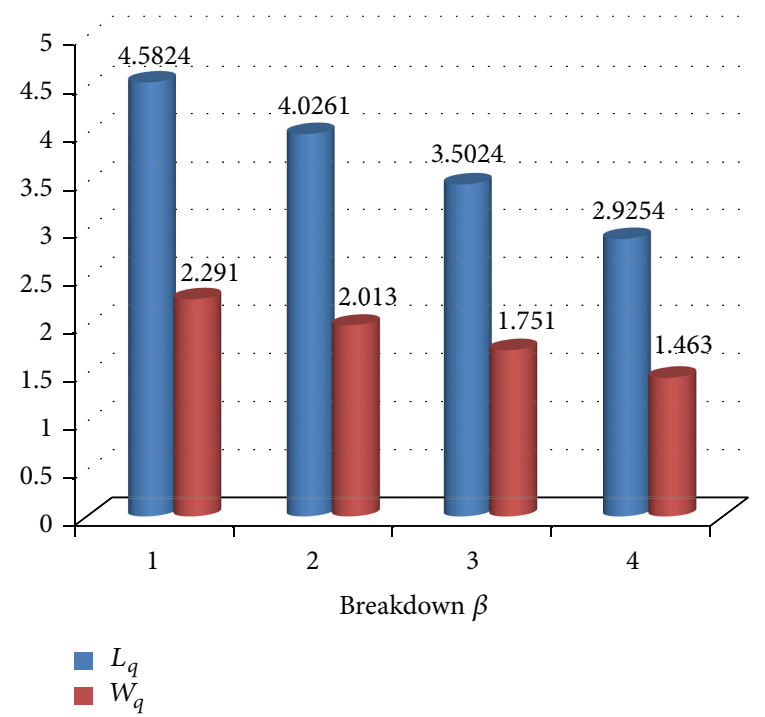

Figure 12: Effect of $\psi=12$ and $\beta=1,2,3$, and 4 on the mean queue size $L_{q}$ and mean waiting time $W_{q}$. The figure explains that as the average length of the queue $L_{q}$ decreases, owing to customers reneging from the queue, the average waiting time $W_{q}$ also decreases.

\section{Acknowledgments}

Authors would like to express appreciation to the anonymous reviewers and the editor Antonina Pirrotta for their very helpful comments that improved the paper.

\section{References}

[1] F. A. Haight, "Queueing with balking," Biometrika, vol. 44, no. 3-4, pp. 360-369, 1957.

[2] F. A. Haight, "Queueing with reneging," Metrika, vol. 2, no. 1, pp. 186-197, 1959.

[3] D. Y. Barrer, "Queuing with impatient customers and ordered service," Operations Research, vol. 5, pp. 650-656, 1957.

[4] A. Montazer-Haghighi, J. Medhi, and S. G. Mohanty, "On a multiserver Markovian queueing system with balking and reneging," Computers \& Operations Research, vol. 13, no. 4, pp. 421-425, 1986.

[5] B. Doshi, "Analysis of a two phase queueing system with general service times," Operations Research Letters, vol. 10, no. 5, pp. 265-272, 1991.

[6] G. Choudhury, "An $\mathrm{M}^{x} / \mathrm{G} / 1$ queueing system with a setup period and a vacation period," Queueing Systems, vol. 36, no. 1-3, pp. 23-38, 2000.

[7] K. C. Madan, "On a single server queue with two-stage heterogeneous service and binomial schedule server vacations," The Egyptian Statistical Journal, vol. 40, no. 1, pp. 39-55, 2000.

[8] J. Bae, S. Kim, and E. Y. Lee, "The virtual waiting time of the M/G/1 queue with impatient customers," Queueing Systems, vol. 38, no. 4, pp. 485-494, 2001.

[9] B. Krishna Kumar, A. Vijayakumar, and D. Arivudainambi, "An $M / G / 1$ retrial queueing system with two-phase service and preemptive resume," Annals of Operations Research, vol. 113, pp. 61-79, 2002.

[10] K. C. Madan, W. Abu-Dayyeh, and F. Taiyyan, "A two server queue with Bernoulli schedules and a single vacation policy," 
Applied Mathematics and Computation, vol. 145, no. 1, pp. 5971, 2003.

[11] G. Choudhury, "A batch arrival queueing system with an additional service channel," International Journal of Information and Management Sciences, vol. 14, no. 2, pp. 17-30, 2003.

[12] K. C. Madan and A. Z. Abu Al-Rub, "On a single server queue with optional phase type server vacations based on exhaustive deterministic service and a single vacation policy," Applied Mathematics and Computation, vol. 149, no. 3, pp. 723-734, 2004.

[13] K. C. Madan and G. Chodhury, "An $\mathrm{M}^{[x]} / \mathrm{G} / 1$ queue with Bernoulli vacation schedule under restricted admissibility policy," Sankhaya, vol. 66, pp. 172-193, 2004.

[14] G. Choudhury and K. C. Madan, "A two-stage batch arrival queueing system with a modified Bernoulli schedule vacation under N-policy," Mathematical and Computer Modelling, vol. 42, no. 1-2, pp. 71-85, 2005.

[15] S. H. Chang and T. Takine, "Factorization and stochastic decomposition properties in bulk queues with generalized vacations," Queueing Systems, vol. 50, no. 2-3, pp. 165-183, 2005.

[16] E. Altman and U. Yechiali, "Analysis of customers' impatience in queues with server vacations," Queueing Systems: Theory and Applications, vol. 52, no. 4, pp. 261-279, 2006.

[17] E. Altman and U. Yechiali, "Infinite-server queues with system's additional tasks and impatient customers," Probability in the Engineering and Informational Sciences, vol. 22, no. 4, pp. 477493, 2008.

[18] R. Kumar and S. K. Sharma, "An M/M/1/N Queueing model with retention of reneged customers and balking," The American Journal of Operations Research, vol. 2, no. 1, pp. 1-5, 2012.

[19] F. A. Maraghi, K. C. Madan, and K. Darby-Dowman, "Bernoulli schedule vacation queue with batch arrivals and random system breakdowns having general repair time distribution," International Journal of Operational Research, vol. 7, no. 2, pp. 240-256, 2010.

[20] R. Kumar and S. K. Sharma, "A Markovian feedback queue with retention of reneged customers and balking," Advanced Modeling and Optimization, vol. 14, no. 3, pp. 681-688, 2012.

[21] M. Baruah, K. C. Madan, and T. Eldabi, "A two stage batch arrival queue with reneging during vacation and breakdown periods," The American Journal of Operations Research, vol. 3, pp. 570-580, 2013.

[22] R. Kumar and S. K. Sharma, "A Markovian multi-server queuing model with retention of reneged customers and balking," International Journal of Operations Research, vol. 20, no. 4, pp. 427-438, 2014. 


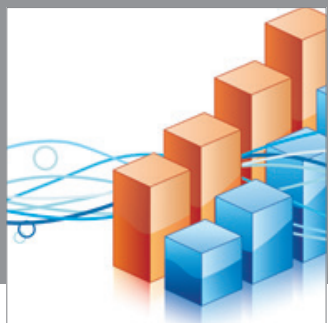

Advances in

Operations Research

mansans

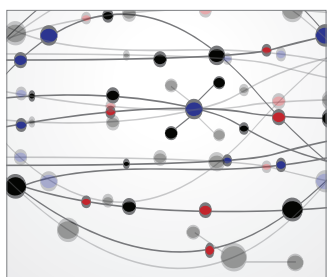

The Scientific World Journal
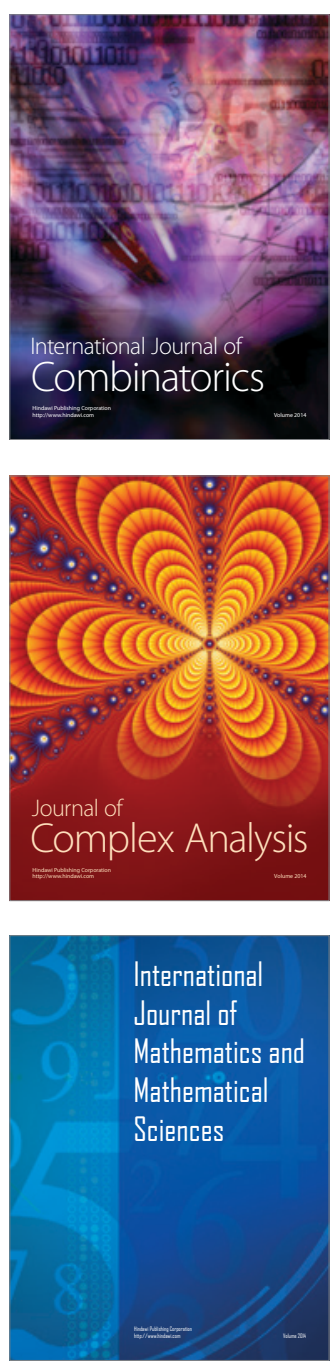
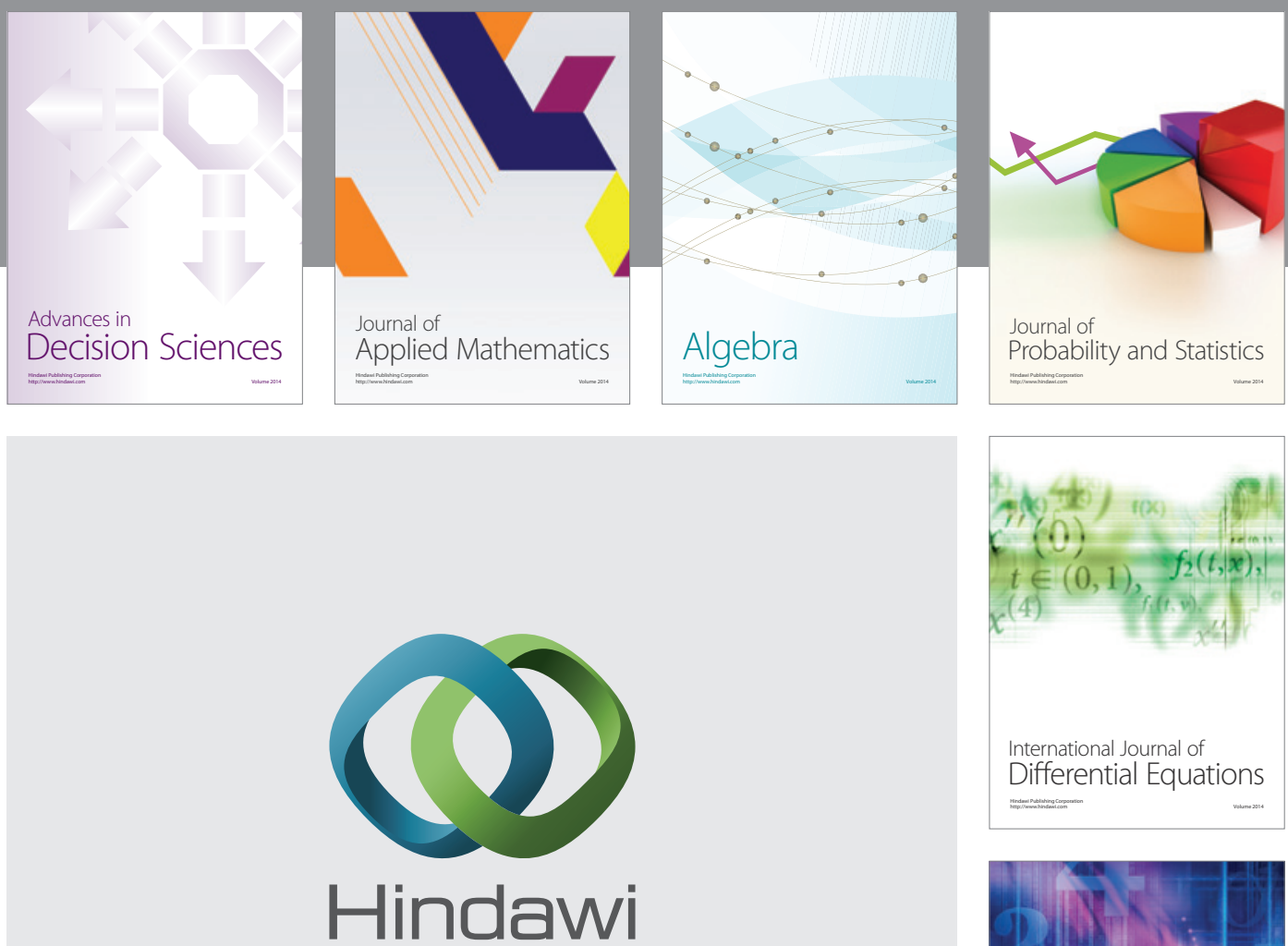

Submit your manuscripts at http://www.hindawi.com
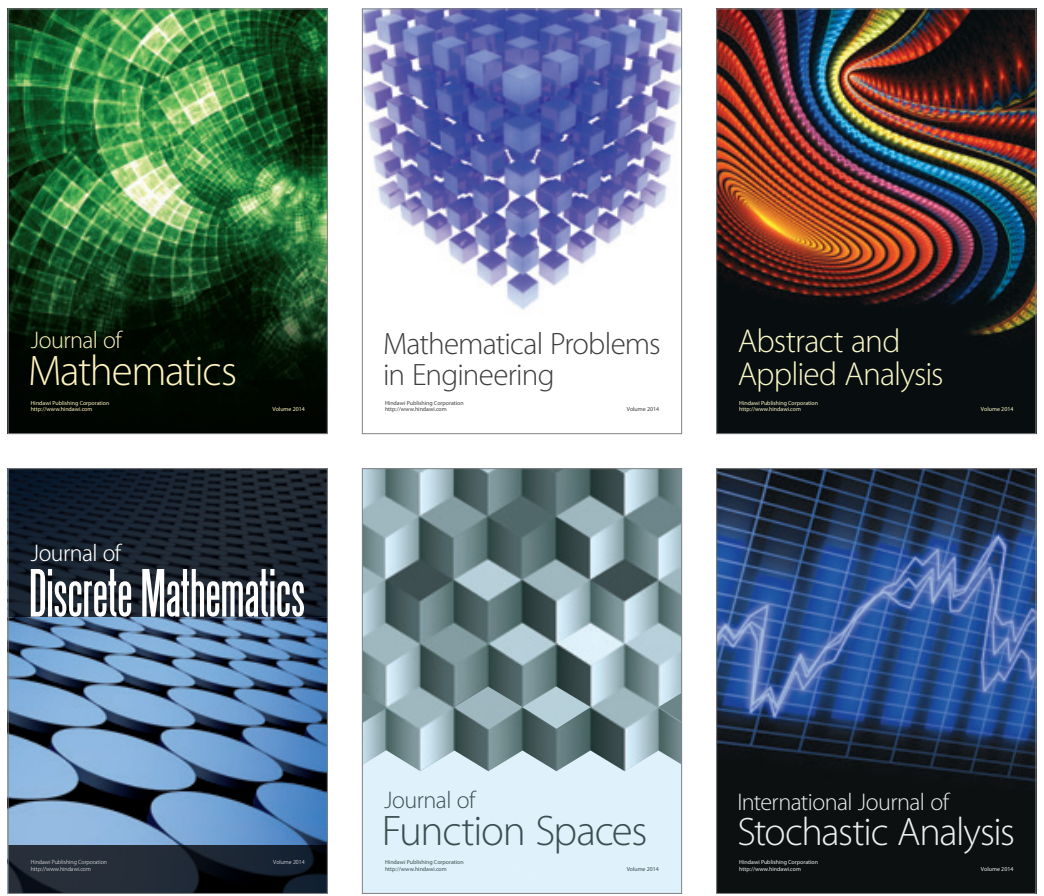

Journal of

Function Spaces

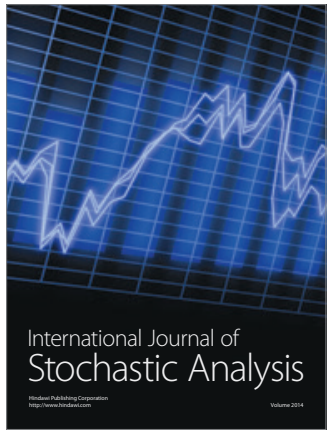

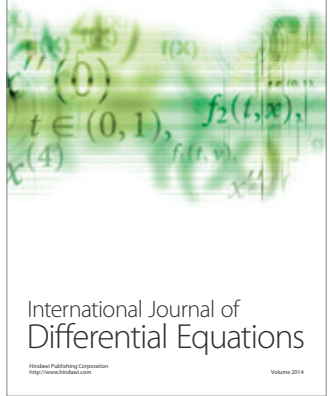
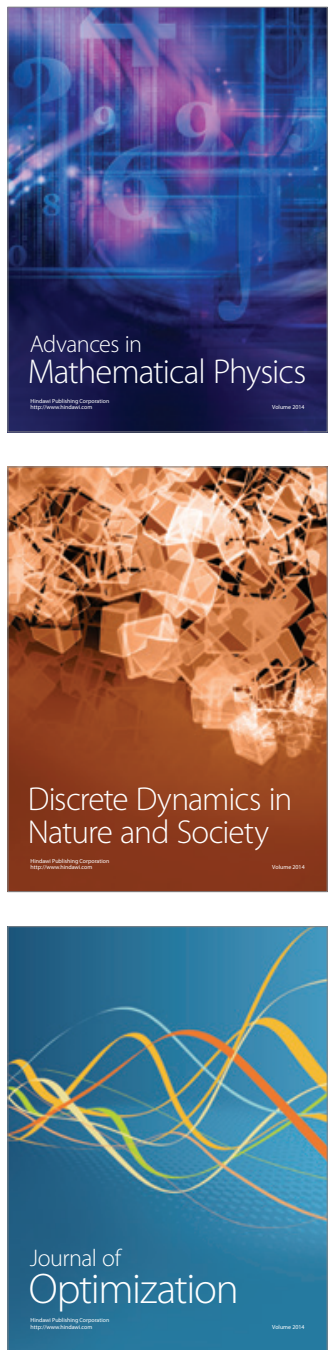